\title{
Tuning Variable Selection Procedures by Adding
}

\section{Noise}

\author{
Xiaohui Luo, ${ }^{*}$ Leonard A. Stefanski and Dennis D. Boos ${ }^{\dagger}$
}

\begin{abstract}
Many variable selection methods for linear regression depend critically on tuning parameters that control the performance of the method, e.g., "entry" and "stay" significance levels in forward and backward selection. However, most methods do not adapt the tuning parameters to particular data sets. We propose a general strategy for adapting variable selection tuning parameters that effectively estimates the tuning parameters so that the selection method avoids overfitting and underfitting. The strategy is based on the principle that underfitting and overfitting can be directly observed in estimates of the error variance after 1) adding controlled amounts of additional independent noise to the response variable and 2) then running a variable selection method. It is related to the simulation technique SIMEX found in the measurement error literature. We focus on forward selection because of its simplicity and ability to handle large numbers of explanatory variables. Monte Carlo studies show that the new method compares favorably with established methods.
\end{abstract}

*Xiaohui Luo is Biometrician, Clinical Biostatistics, Merck Research Laboratories, Rahway, NJ 07065-0900 (Email: edmund_luo@merck.com).

${ }^{\dagger}$ Leonard A. Stefanski and Dennis D. Boos are Professors, Department of Statistics, North Carolina State University, Raleigh, NC 27695-8203 (E-mail: stefansk@stat.ncsu.edu, boos@stat.ncsu.edu). 
KEY WORDS: AIC; BIC; Forward selection; Mallows Cp; Model selection; Regression; SIMEX.

\section{INTRODUCTION}

Common variable selection methods such as stepwise, forward and backward selection, and generalizations of Mallows $C_{p}$ (e.g., Atkinson, 1980) depend on tuning parameters that control the selection procedure, e.g., entry/stay significance levels for stepwise, forward and backward selection, and a penalty factor for generalized Mallows $C_{p}$. Depending on the choice of tuning parameter, the selection procedure may tend to underfit or overfit the model by selecting too few or too many variables. For example, the default significance level to enter in the SAS forward selection procedure is $\alpha_{\text {Enter }}=0.50$ and is known to result in overfitting. Conversely, taking $\alpha_{\text {Enter }}$ too small will prevent important predictors from entering the model. Other variable selection procedures have similar features. Atkinson's (1980) generalization of Mallows $C_{p}$ chooses the subset of variables that minimizes a penalized log-likelihood criterion. The tuning parameter is the penalty for adding an additional variable to the model. If this penalty is negligible, then unimportant variables will enter the model at a high rate. If it is too large, then important variables will be restricted from entering the chosen model.

Some studies provide general guidelines for selecting tuning parameters. For example, based on simulation results, Atkinson (1980) and Zhang (1992) suggested that the penalty factor for Atkinson's generalized Mallows $C_{p}$ statistic should be chosen between 1.5 and 6 (Mallows $C_{p}$ is obtained when the penalty factor is set equal to 2). Forward selection is well discussed in many regression texts, e.g., Draper and Smith (1981). However, there has been little, if any, work 
on the use of data-dependent tuning parameters for forward selection and generalized $C_{p}$. The research described in this paper addresses this void. We introduce a generally applicable, simulationbased method for choosing tuning parameters in variable selection procedures. The new method exploits the fact that, when coupled with a variable selection procedure, both underfitting and overfitting result in biased estimates of the error variance. The basic idea is to add controlled amounts of random error to the response variable and tune the model selection procedure to estimate the new error variance in an unbiased way. The method is related to the SIMEX (simulation extrapolation) method used in the measurement error literature to estimate and reduce bias (Cook and Stefanski, 1994; Stefanski and Cook, 1995; Carroll et al., 1995). In model selection contexts Breiman (1992) adds random error to the response variable to estimate model error (see definition below), and Ye (1998) and Shen and Ye (2002) use it to estimate model degrees of freedom.

Following a general introduction and discussion of variable selection in linear models in Section 2., the tuning strategy is outlined in general terms and in detail for forward selection in Section 3. The method is extensively studied via simulation in Section 4., and further illustrated by application to two data sets in Section 5 .

\section{VARIABLE SELECTION IN LINEAR REGRESSION}

Consider the linear regression model

$$
\boldsymbol{Y}=\boldsymbol{\mu}+\boldsymbol{\epsilon}=\boldsymbol{X} \boldsymbol{\beta}+\boldsymbol{\epsilon}
$$

where $\boldsymbol{Y}=\left(Y_{1}, Y_{2}, \ldots, Y_{n}\right)^{T}$ is a vector of dependent variables, $\boldsymbol{\mu}=\mathrm{E}(\boldsymbol{Y})=\boldsymbol{X} \boldsymbol{\beta}, \boldsymbol{X}$ is a full-rank design matrix with $n$ observations and $p_{\text {Max }}$ predictors, $\boldsymbol{\epsilon} \sim N\left(\mathbf{0}, \sigma^{2} \boldsymbol{I}_{\boldsymbol{n}}\right)$ with $\sigma^{2}$ unknown and $\boldsymbol{I}_{n}$ the $n \times n$ identity matrix, and $\boldsymbol{\beta}$ is the coefficient vector possibly with some elements equal to 0 . 
Henceforth, predictors with nonzero coefficients are called important, those with zero coefficients are unimportant. If the rows of $\boldsymbol{X}$ are random, then we are thinking of conditional analyses holding $\boldsymbol{X}$ fixed.

Two common objectives of variable selection are interpretation and prediction. When statistical modeling is an adjunct to the development and testing of substantive theoretical models, the variables that enter the model are important because they often give insight to and sometimes guide the process of theoretical model development. For such applications of variable selection the ability to distinguish between important and unimportant variables is paramount. This aspect of variable selection is not as crucial when prediction is the sole or primary objective of modeling. For prediction, the utility of the selected model is often evaluated by prediction error (PE), defined as

$$
\mathrm{PE}=\mathrm{E}\left(\boldsymbol{Y}^{n e w}-\widehat{\boldsymbol{Y}}\right)^{T}\left(\boldsymbol{Y}^{n e w}-\widehat{\boldsymbol{Y}}\right)=\mathrm{ME}+n \sigma^{2}
$$

where the expectation is taken only with respect to $\boldsymbol{Y}^{\text {new }}$, and

$$
\mathrm{ME}=(\widehat{\boldsymbol{Y}}-\boldsymbol{\mu})^{T}(\widehat{\boldsymbol{Y}}-\boldsymbol{\mu})
$$

where $\widehat{\boldsymbol{Y}}$ is the least squares estimate of $\boldsymbol{Y}, \boldsymbol{Y}^{\text {new }}$ is a random vector independently and identically distributed as $\boldsymbol{Y}$, and ME denotes the model error. The expected prediction error is thus determined by the expected model error given by,

$$
\mathrm{E}(\mathrm{ME})=(\mathrm{E}(\widehat{\boldsymbol{Y}})-\boldsymbol{\mu})^{T}(\mathrm{E}(\widehat{\boldsymbol{Y}})-\boldsymbol{\mu})+p \sigma^{2}
$$

where $p$ is the model size. Because $\mathrm{E}(\mathrm{ME})$ depends on bias and variance, variable selection for the 
purpose of prediction entails a bias-variance tradeoff. Entering more variables generally reduces bias, but inflates variance.

Many model selection criteria are based on estimating statistical information such as the Akaike Information Criterion (AIC) and the Bayesian Information Criterion (BIC). The key idea behind an information criterion is to penalize the likelihood for the model complexity and select a model by maximizing the penalized likelihood criterion. Under linear regression with normal errors, AIC is asymptotically equivalent to Mallows $C_{p}$ statistic, which is a special case of Atkinson's (1980) generalized $C_{p}$ statistic, given by

$$
\operatorname{GMC}_{\eta}(J)=\frac{\operatorname{SSE}(J)}{\sigma^{2}}-n+\eta p_{J}
$$

where $J$ is a subset model, $p_{J}$ is the number of predictors in $J, \operatorname{SSE}(J)$ is the residual sum of squares of $J, \eta$ is the penalty factor for model complexity, and in practice $\sigma^{2}$ is usually estimated by $\widehat{\sigma}_{F}^{2}$, the residual mean squares from the full model. For given $\eta$, a model is selected by minimizing $\operatorname{GMC}_{\eta}(J)$ over all the subset models. But different models may be selected due to different choices of penalty factors.

Mallows $C_{p}(\eta=2)$ is used widely and abused as well. It was pointed out by Mallows (1973) and reemphasized by Mallows (1995) that $\mathrm{E}(\mathrm{PE})=\mathrm{E}\left(\widehat{\sigma}^{2} C_{p}\right)$ if the additive error is independently distributed with mean 0 and variance $\sigma^{2}, \widehat{\sigma}^{2}$ is an unbiased estimator of $\sigma^{2}$, and the model is selected independently of the data under study. But the last assumption is usually conveniently overlooked in practice. Hence selecting a model by minimizing $C_{p}$ tends to overfit, a fact which is manifest in the Monte Carlo study in Section 4.

Our goal is to estimate $\boldsymbol{\beta}$ and predict $\boldsymbol{Y}^{\text {new }}$. If we fit a subset model $J$, then the usual residual 
mean squares is given by

$$
\operatorname{MSE}(J)=\frac{\boldsymbol{Y}^{T}\left(\boldsymbol{I}_{n}-\boldsymbol{P}_{J}\right) \boldsymbol{Y}}{n-p_{J}}
$$

where $\boldsymbol{I}_{n}$ is an $n \times n$ identity matrix, and $\boldsymbol{P}_{J}=\boldsymbol{X}_{J}\left(\boldsymbol{X}_{J}^{T} \boldsymbol{X}_{J}\right)^{-1} \boldsymbol{X}_{J}^{T}$, the projection matrix of $\boldsymbol{X}_{J}$.

If $J$ contains all the important variables, $\left(n-p_{J}\right) \mathrm{MSE} / \sigma^{2}$ follows a $\chi_{n-p_{J}}^{2}$ distribution and hence $\mathrm{E}(\mathrm{MSE})=\sigma^{2}$; we call such models inclusive. Otherwise, $\left(n-p_{J}\right) \mathrm{MSE} / \sigma^{2}$ follows a noncentral $\chi_{n-p_{J}}^{2}$ distribution with $\mathrm{E}(\mathrm{MSE})>\sigma^{2}$; such models are called non-inclusive. If the coefficients of the important variables are not too small and the predictors are uncorrelated, then we will have a very good separation between inclusive models and non-inclusive models. Consequently we can find the true model by intersecting all the inclusive models.

For example, suppose that there are six important variables (with coefficients all equal) among twelve uncorrelated predictors, and that the error follows a standard normal distribution. The common value of the coefficients is chosen so that the theoretical $R^{2}=0.75$. In Figure 1 (a), the MSE of each subset model is plotted versus its model size. All the inclusive models appear at the bottom of the plot. For model sizes less than six, there is no separation between the models because all the models are non-inclusive. In Figure 1 (b), the best non-inclusive models (giving minimum MSE) at each model size $\geq 4$ are plotted and connected, and all the inclusive models are plotted as well. In addition a horizontal line at $\widehat{\sigma}_{F}^{2}$ is drawn as a baseline. We see that the models are well separated. But if the predictors are correlated or some of the coefficients are tiny, then the noncentral parameter of the $\chi^{2}$ distribution will be very small, and consequently it is more difficult to distinguish inclusive models from non-inclusive models.

One common approach to model selection is to first identify the model maximizing $R^{2}$ at each model size, and then compare MSE of the candidate models with the baseline $\widehat{\sigma}_{F}^{2}$. A model is classified as acceptable if it and those with larger model size have MSE smaller than or equal to 


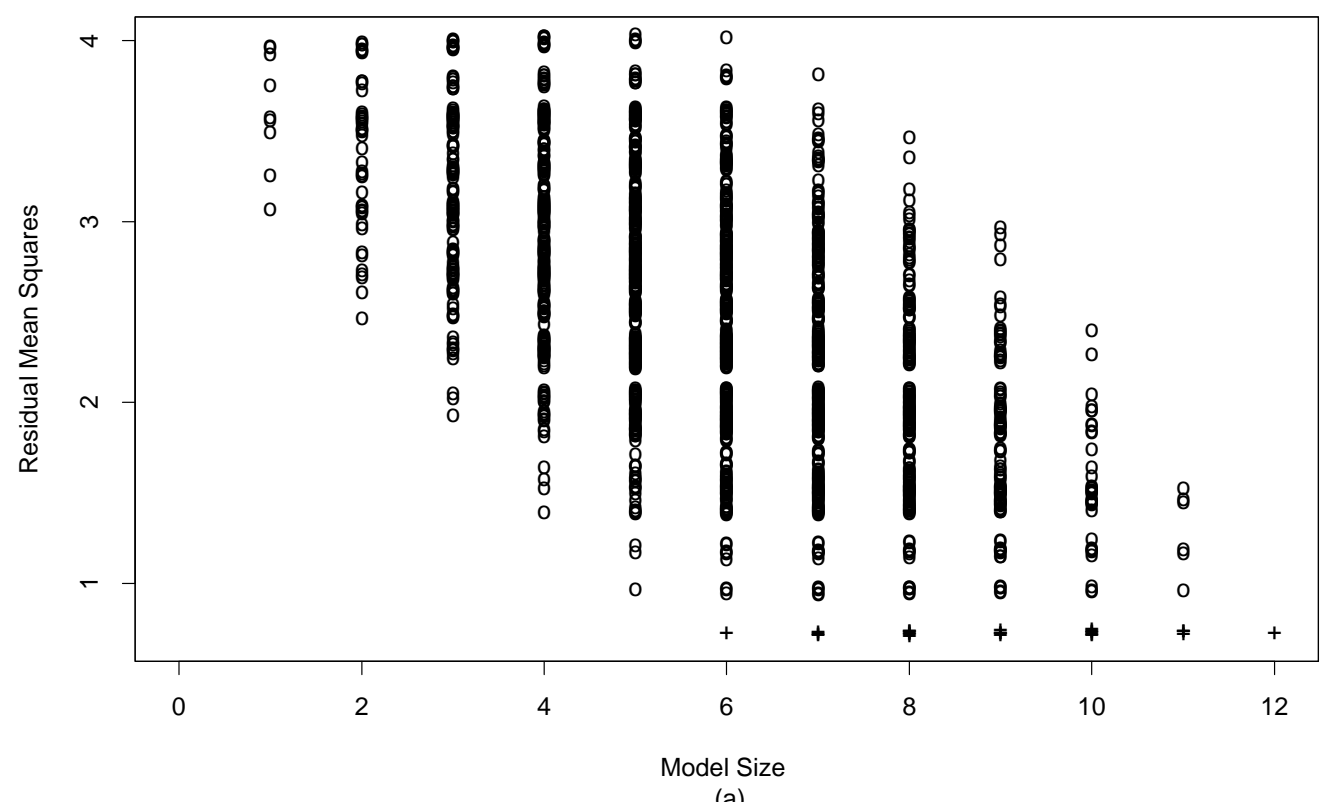

(a)

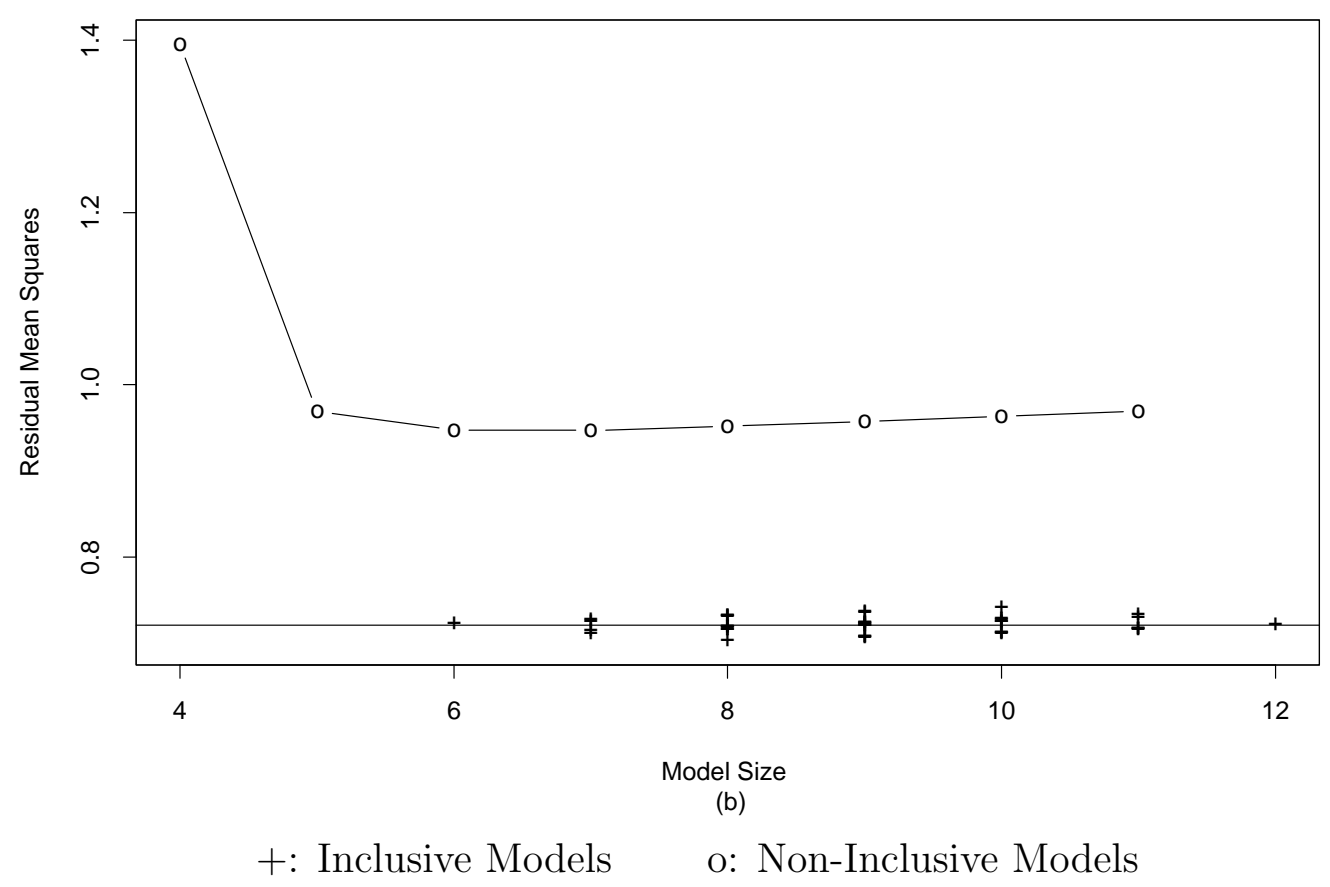

Figure 1: Inclusive Models and Non-Inclusive Models with uncorrelated predictors. Figure (b) is an enlargement of the lower third of Figure (a) and clearly shows the separation between the inclusive and non-inclusive models. 
the baseline. The model with the smallest size among the acceptable candidate models is then selected. This approach is equivalent to applying the same rule on Mallows $C_{p}$ but using model size as a baseline, which views Mallows $C_{p}$ as an estimate of the model size (Mallows 1995). For subset model $J$,

$$
\begin{aligned}
& \qquad C_{p}=\frac{\operatorname{SSE}(J)}{\widehat{\sigma}_{F}^{2}}-\left(n-2 p_{J}\right)=\left(n-p_{J}\right)\left(\frac{\operatorname{MSE}(J)}{\widehat{\sigma}_{F}^{2}}-1\right)+p_{J} \\
& \text { and then, } \quad C_{p} \leq p_{J} \Longleftrightarrow \operatorname{MSE}(J) \leq \widehat{\sigma}_{F}^{2}
\end{aligned}
$$

We call this approach intelligent $C_{p}$ and denote it by $I C_{p}$ to distinguish it from the minimum $C_{p}$ method that selects the model with the smallest $C_{p}$ value. The $I C_{p}$ method is illustrated in Figure 2. In this plot, the models are pre-selected by maximizing $R^{2}$ at each model size. A horizontal line at $\widehat{\sigma}_{F}^{2}$ is drawn as our baseline. MSE decreases as model size decreases from twelve to eight, while it increases as model size decreases from eight to six. All the pre-selected models with model size greater than six are acceptable, but the model with six predictors is not. Therefore, the model with seven predictors is selected.

For simplicity and also to handle models with large numbers of candidate variables (large $p_{\text {Max }}$ ), we have chosen to develop and apply our simulation-based variable selection method in the context of forward selection. For comparison purposes we also included in our simulation studies a modified $C_{p}$ method applied to the candidate models from forward selection (instead of by maximizing $\left.R^{2}\right)$, calling it forward-restricted intelligent $C_{p}\left(I C_{p} / F R\right)$. In addition we include Breimans little bootstrap (LB) restricted to forward selection with the parameters suggested by Breiman (1992). 


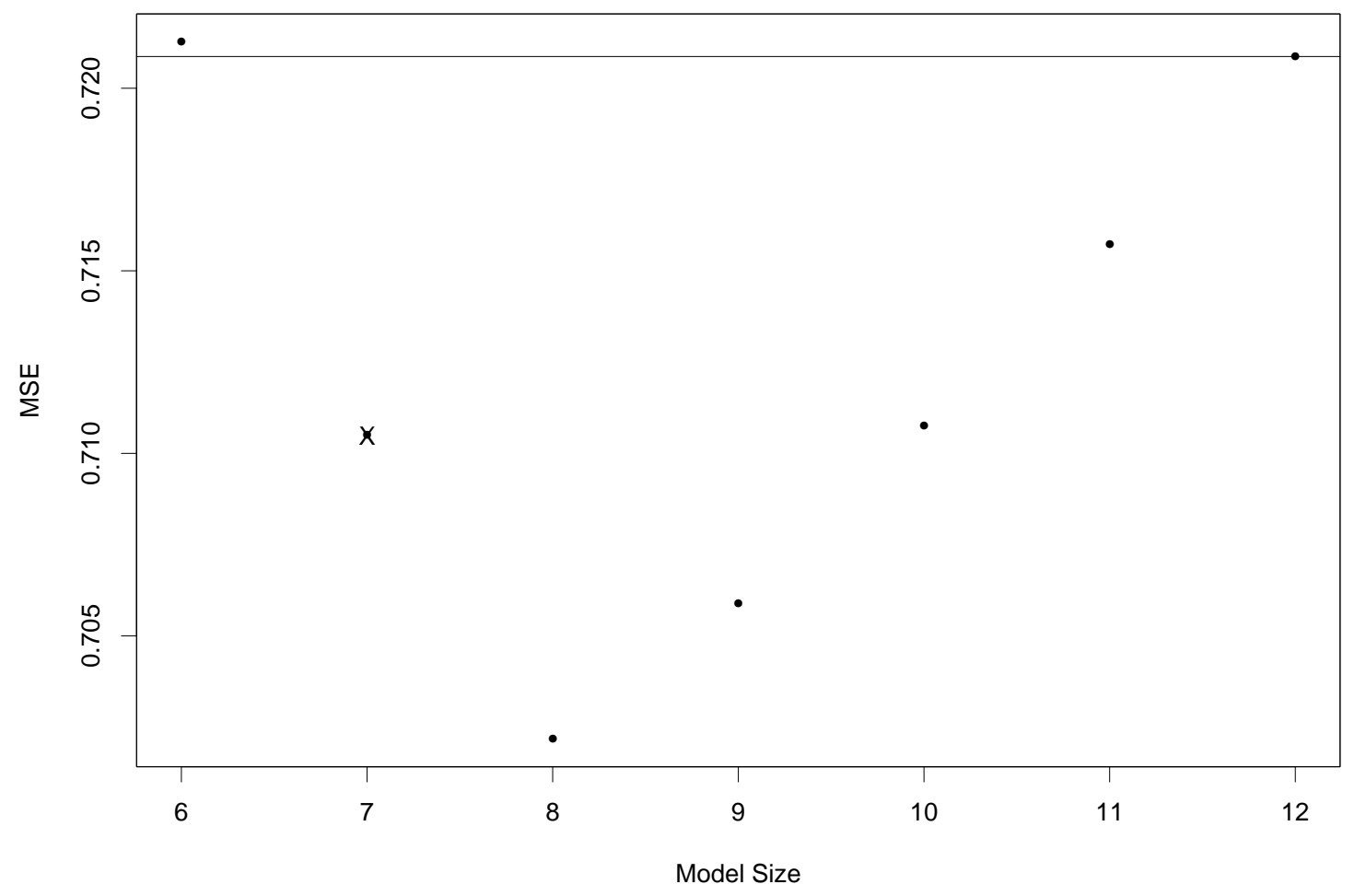

Figure 2: Illustration of model selection with $\mathrm{IC}_{p}$. The selected model is indicated by an $\times$. 


\section{TUNING VARIABLE SELECTION PROCEDURES BY ADDING NOISE}

Selection of a set of regressors induces a partition of the total variation in $\boldsymbol{Y}$ into regression (SSR) and error (SSE) components. A liberal variable selection method over-selects variables resulting in an inflated SSR and a deflated SSE. A conservative variable selection method under-selects variables thereby inflating SSE and deflating SSR. Although degrees-of-freedom corrections alleviate these biases somewhat, they still are manifest in mean squares (MSR and MSE).

The latter claim is somewhat easier to explain for the case of liberal methods. Suppose that a method is liberal, i.e., it tends to misclassify unimportant variables thereby including them in the regression model. It follows that the number of variables in the chosen model tends to exceed the number of important variables. The fact that variable selection methods generally identify the best-fitting subset of variables of a given size, implies that the chosen model tends to have a mean squared error that is as small as or smaller than the minimum MSE of all of the inclusive models of the chosen model's size. Any particular inclusive model admits an unbiased estimator of $\sigma^{2}$. However, the minimum of a set of unbiased estimators will generally be biased low, and thus the MSE of a model identified by a liberal variable selection procedure tends to be biased low.

If the method is conservative, then the chosen model tends not to be inclusive. The claim that in such cases the MSE is biased high is based on the fact that for the case of random regressors, $\mathrm{E}\left\{\operatorname{Var}\left(\boldsymbol{Y} \mid S_{1}\right)\right\} \geq \mathrm{E}\left\{\operatorname{Var}\left(\boldsymbol{Y} \mid S_{2}\right)\right\}$ for subsets of regressor variables $S_{1} \subseteq S_{2}$ with strict inequality

when all important variables are in $S_{2}$ but not all are in $S_{1}$. However, in this case the fact that variable selection methods generally identify the best-fitting subset of variables of a given size works to offset the increase in MSE due to regression on a non-inclusive set of predictors. The net effect of these two biasing factors in finite samples is not easily analyzed and depends to a great extent on the magnitude of the regression coefficients of the omitted predictors and sample size. 
In summary, the biasing effects of liberal methods are fairly well established and understood whereas those of conservative methods are more dependent on particulars of the data set under analysis. The trick is to exploit this information to determine the value of the tuning parameter that admits the least-biased, liberal method. This can only be accomplished if we have a means for identifying liberal choices of tuning parameters. Here is where we borrow ideas from the measurement error literature. Simulation extrapolation (SIMEX) is a technique for studying the biasing effects of measurement error (Cook and Stefanski, 1994; Stefanski and Cook, 1995; Carroll et al., 1995). It works by adding additional measurement error to the data, and studying the effects this has on statistics calculated from remeasured data. It is in this spirit that we apply SIMEX ideas to the variable selection problem.

Assume the linear model (1) for the response variable $\boldsymbol{Y}$. Consider the remeasured variable

$$
\boldsymbol{Y}^{*}=\boldsymbol{Y}+\tau \sqrt{\lambda} \boldsymbol{Z}
$$

where $\boldsymbol{Z}$ is a $\mathrm{N}\left(\mathbf{0}, \boldsymbol{I}_{n}\right)$ pseudo-random vector, and $\lambda>0$ and $\tau>0$ control the variance of the added errors. The obvious over-parameterization reflects the different roles played by $\lambda$ (as the scale) and $\tau$ (as the unit) in control of the added errors. The remeasured response variable has the property that

$$
\mathrm{E}\left(\boldsymbol{Y}^{*} \mid \boldsymbol{X}\right)=\mathrm{E}(\boldsymbol{Y} \mid \boldsymbol{X}), \quad \text { and } \quad \operatorname{Var}\left(\boldsymbol{Y}^{*} \mid \boldsymbol{X}\right)=\operatorname{Var}(\boldsymbol{Y} \mid \boldsymbol{X})+\tau^{2} \lambda \boldsymbol{I}_{n}
$$

i.e., the conditional expectations of $\boldsymbol{Y}$ and $\boldsymbol{Y}^{*}$ given $\boldsymbol{X}$ are identical, and the variance of $\boldsymbol{Y}^{*}$ given $\boldsymbol{X}$ exceeds the variance of $\boldsymbol{Y}$ given $\boldsymbol{X}$ by a known, controlled amount $\left(\tau^{2} \lambda\right)$. Note that these conditional moment results do not depend on any assumed regression model. 
Now suppose that the remeasured data $\left(Y_{i}^{*}, X_{i}\right), i=1, \ldots, n$ are passed to a tuning parametercontrolled variable selection procedure, e.g., forward selection. If the entry significance level is set too high (so that forward selection is liberal), the expected increase (relative to the true data) in MSE of $\tau^{2} \lambda$ will not be fully realized. A liberal method will tend to select unimportant predictors, thereby biasing MSE low for the reasons noted previously. In effect the liberal method fits (some of) the noise. We can see this in the statistics generated from remeasured data by generating remeasured data sets with different values of $\lambda$ ( $\tau$ remains fixed). The mean squared errors from the models found by forward selection with a liberal value of the entry significance level, and for different values of $\lambda$, when plotted against $\lambda$ will tend to have slope less than $\tau^{2}$. Alternatively, if in the forward selection, the entry significance level was such that there was no bias in the MSEs of the forward-selected models, then the MSEs of the remeasured data sets should increase linearly in $\lambda$, with slope equal to $\tau^{2}$.

The previous argument depends critically on the selection bias that results from choosing the best model from a set of inclusive models. Therefore it breaks down in the extreme cases for which the significance level to enter is equal to 1.0 or 0.0 . In the former case, forward selection enters every variable, and provided the model is full rank, the MSE of the full model will have expectation that increases exactly linearly in $\lambda$ with slope $\tau^{2}$. In the latter case forward selection excludes every variable, and the MSE of the null model will also have expectation that increases exactly linearly in $\lambda$ with slope $\tau^{2}$.

We can now describe our tuning strategy for forward selection as an algorithm.

1. Generate independent remeasured data sets for each of a grid of $\lambda$ 's:

$$
0<\lambda_{1}<\cdots<\lambda_{n_{\lambda}} \leq 4
$$

2. For a grid of entry significance levels $\alpha \in[0,1]$, run forward selection on the remeasured data 
and calculate the average MSE (over identically distributed remeasured data sets); call these $\overline{\operatorname{MSE}}\left(\lambda_{k}, \alpha\right), k=1, \ldots n_{\lambda}$. (Note: the remeasured data sets are the same for all $\alpha$ but different for different values of $\lambda$.)

3. For each $\alpha$ fit a line to the pairs $\left\{\lambda_{k}, \overline{\operatorname{MSE}}\left(\lambda_{k}, \alpha\right), k=1, \ldots n_{\lambda}\right\}$ and call the resulting slope $\widehat{\gamma}(\alpha)$.

4. Our target, or "tuned" entry significance level is the value of $\alpha$ in the open interval $(0,1)$ for which $\widehat{\gamma}(\alpha)=\tau^{2}$.

The above algorithm works because for $\alpha \in(0,1)$ the slope $\widehat{\gamma}(\alpha)$ will be less than $\tau^{2}$ for $\alpha$ too large and greater than $\tau^{2}$ for $\alpha$ too small.

In practice we must choose the grid of $\alpha$ values along with the grid of $\lambda$ values, and we must deal with finite sample and Monte Carlo variation in the estimated slopes when identifying the interior value of $\alpha$ for which $\widehat{\gamma}(\alpha)=\tau^{2}$. We now describe the specifics of the implementation used in our simulation study.

First, the added variance multiplier, $\tau^{2}$, should be appropriately scaled and we took $\tau^{2}=\widehat{\sigma}_{F}^{2}$, the estimate of $\sigma^{2}$ from the fit of the full model, so that the remeasured data are

$$
Y_{i, \lambda_{k}, b}^{*}=Y_{i}+\sqrt{\lambda_{k}} \widehat{\sigma}_{F} Z_{i, \lambda_{k}, b}, \quad i=1,2, \ldots, n, k=1, \ldots, n_{\lambda}, b=1, \ldots, B
$$

where $Z_{i, \lambda_{k}, b}$ independent and identically distributed standard normal random variables, independent of the original data. Note that the same contaminated data sets are used for each $\alpha$, but are independent for different $\lambda$. The remeasured data are passed to forward selection with entry level $\alpha$ and the average (over $b=1, \ldots, B)$ mean squared errors, $\overline{\operatorname{MSE}}\left(\lambda_{k}, \alpha\right)$ is computed.

We calculate the least squares slope, $\widehat{\gamma}(\alpha)$, of the line fit to $\left\{\lambda_{k}, \overline{\operatorname{MSE}}\left(\lambda_{k}, \alpha\right)\right\}$. With $\alpha=1$, 
forward selection will fit the full model for all the contaminated data sets, and $\widehat{\gamma}(1)$ is a consistent estimator of $\widehat{\sigma}_{F}^{2}$ as $\mathrm{B} \rightarrow \infty$. Instead of $\widehat{\sigma}_{F}^{2}$, we use $\widehat{\gamma}(1)$ as a baseline to which other slopes $\widehat{\gamma}(\alpha)$ are compared. This substitution is a type of Monte Carlo swindle. The target tuning parameter $0<\widehat{\alpha}_{*}<1$, should satisfy $\widehat{\gamma}\left(\widehat{\alpha}_{*}\right) \approx \widehat{\gamma}(1)$. Plots of $\widehat{\gamma}(\alpha)$ versus $\alpha$ are informative and should be examined in particular applications. They sometimes reveal a clear, well-defined value of $\widehat{\alpha}_{*}$; and in cases where they do not, the ambiguity usually is a consequence of two or more nearly identically good models corresponding to two or more close values of $\alpha$. In practice all such models should be considered. However, for our Monte Carlo study it was necessary to implement an automated method of identifying a particular $\widehat{\alpha}_{*}$ and the corresponding model. We define $\widehat{\alpha}_{*}$ as the smallest value of $\alpha$ that is greater than $\widehat{\alpha}_{0}$, where

$$
\widehat{\alpha}_{0}=\operatorname{Max}\{\alpha<1: \widehat{\gamma}(\alpha)>\widehat{\gamma}(1) \text { or } \widehat{\gamma}(\alpha) \text { is a local maximum }\}
$$

The defining rule is illustrated in Figure 3, where the models selected are marked by $\oplus$.

A key to our tuning procedure is that after white noise is added to the original data, forward selection should yield a different sequence of candidate models, which helps distinguish the optimal value of $\alpha$. We need to add enough noise in order to introduce sufficient variation among the sequences. But the noise level should not be too high, otherwise $\overline{\operatorname{MSE}}(\lambda, \alpha)$ is not (approximately) linear in $\lambda$. In a pilot study, we set $\lambda=(0.25,0.5,0.75,1,1.5,2,3,4)$ and fitted the simple linear regression of $\overline{\operatorname{MSE}}(\lambda, \alpha)$ on different combinations of the above $\lambda$ 's and found that $\lambda=(0.5,1,1.5,2)$ provides satisfactory results. Note that the MSE from the original data (corresponding to $\lambda=0$ ), is not included in the simple linear regression to estimate the slope. This is because there is no Monte Carlo variation at $\lambda=0$ to smooth out discontinuities in $\overline{\operatorname{MSE}}(0, \alpha)$ as a function of $\alpha$.

Based on our pilot study we decided to take $\mathrm{B}=4000$, so as to guarantee good estimates of 


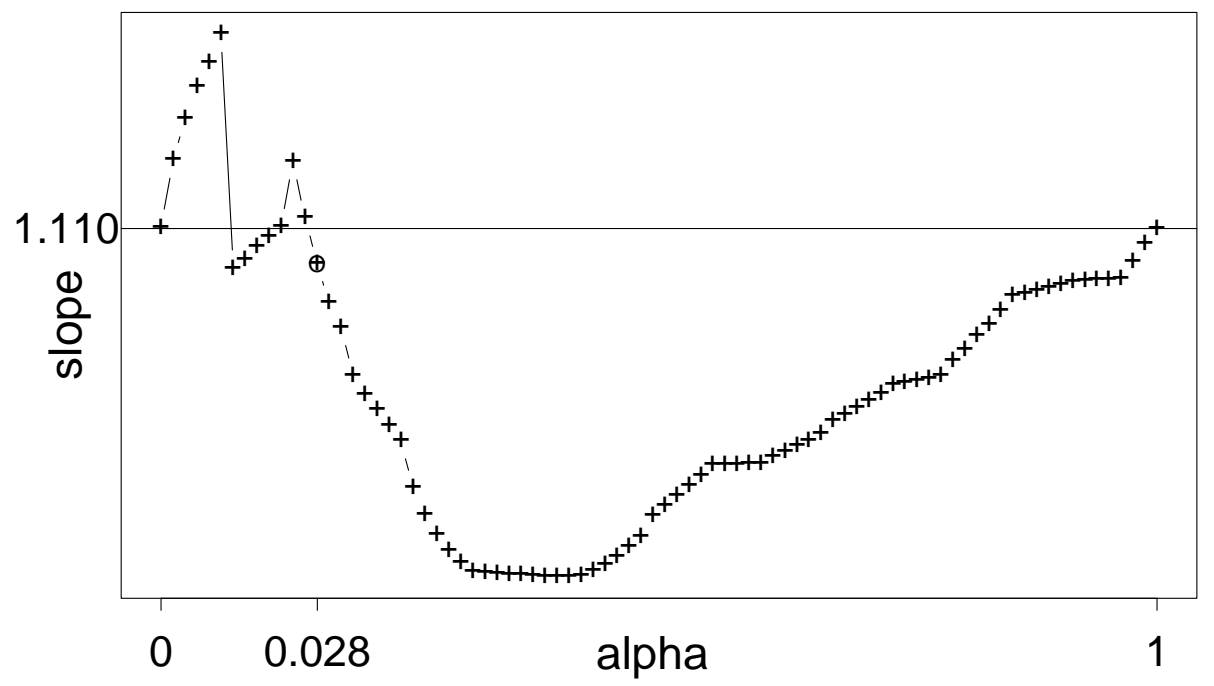

(a)

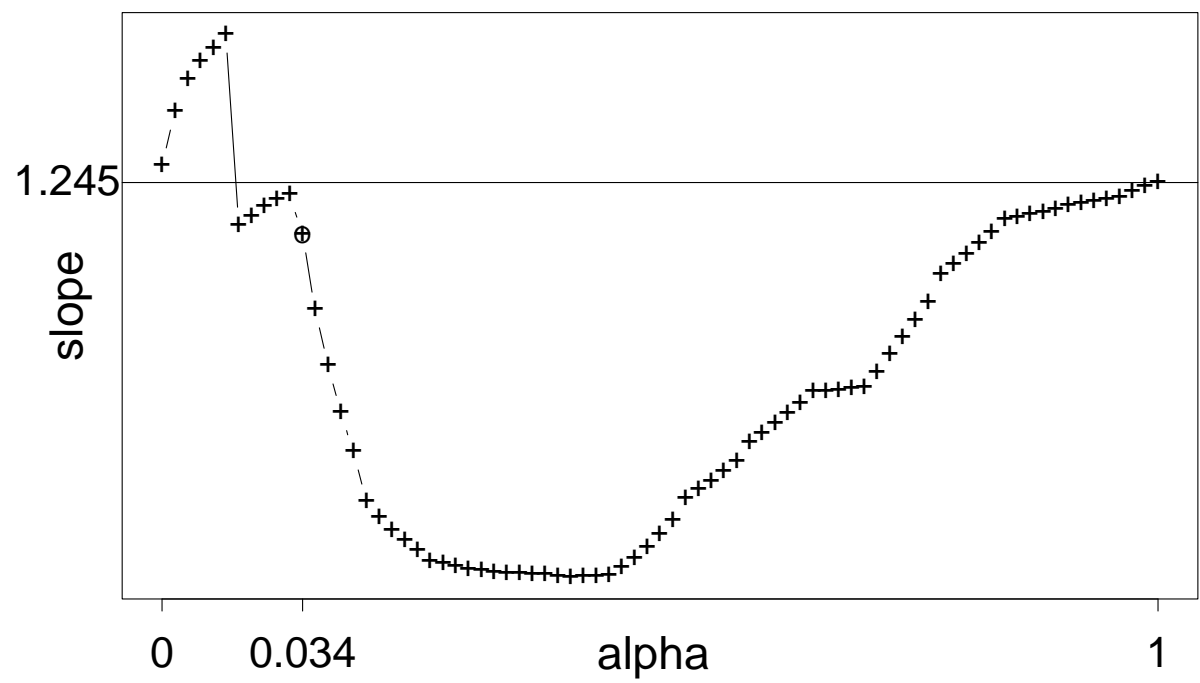

(b)

Figure 3: Demonstration of the Tuning Procedure

(a): $\widehat{\alpha}_{*}$ identified by a crossing of the baseline

(b): $\widehat{\alpha}_{*}$ identified by a local maximum 
slopes. The pilot study also suggested that the grid of $\alpha$ values is important. The finer the grid the better, but this increases the computational burden. We found that no universal set works for all data sets, and we suggest choosing the $\alpha$ 's adaptively based on the real data under study. For a given real data set, the order of the predictors entering the model in forward selection is determined, and so is the significance level associated with each step (more than one predictor can enter the model at one step). Suppose the full model is obtained after $k$ steps $\left(k \leq p_{\max }\right)$, and the associated significance levels are $0<a_{1}<a_{2}<\ldots<a_{k}<1$. Then we chose the $\alpha$ 's as 0 , $\left\{\frac{a_{i}+5 a_{i-1}}{6}, \frac{2 a_{i}+4 a_{i-1}}{6}, \frac{3 a_{i}+3 a_{i-1}}{6}, \frac{4 a_{i}+2 a_{i-1}}{6}, \frac{5 a_{i}+a_{i-1}}{6}, i=1,2, \ldots, k\right\}, \frac{1+2 a_{k}}{3}, \frac{2+a_{k}}{3}$, and 1, where $a_{0}=0$

\section{MONTE CARLO STUDY}

We performed a simulation study designed to compare our proposed method (hereafter called NAMS, which stands for Noise Addition Model Selection), to $I C_{p} / F R$, LB, and Minimum $C_{p}$. Minimum $C_{p}$ is based on all subsets, and $I C_{p} / F R$ and LB are based on forward selection candidate models. In addition, the model with the minimum model error among the forward selection candidate models was identified and designated "Best" (which is the best that a forward selection procedure can do in prediction, though in practice we do not have such information).

\subsection{Simulation Setup}

The setup of the simulation follows closely, and extends somewhat, the setup in Tibshirani and Knight (1999), which is a simplified version of Breiman (1992). In the base simulation each data set contains 21 predictors with $n=50$ or 150 observations. The predictors follow a multivariate normal distribution with mean 0 and covariance between $x_{i}$ and $x_{j}$ equal to $\rho^{|i-j|}$, with $\rho=0,0.3$, and 0.7 , respectively. The design matrices are fixed once generated. We have six different design matrices corresponding to the combinations of $n$ and $\rho$. The coefficients of the important variables 
(those with non-zero coefficient) are generated in two clusters, around $x_{7}$ and $x_{14}$. Their initial values are

$$
\begin{array}{ll}
\beta_{7+j}=(h-j)^{2}, & |j|<h \\
\beta_{14+j}=(h-j)^{2}, & |j|<h
\end{array}
$$

The values $h=1,2,3,4$ are used, resulting in 2,6,10 and 14 non-zero coefficients, respectively. In addition, the null model H0 (all the coefficients are 0) and the full model FULL (with the coefficients generated randomly from Uniform $(0.5,1.5))$ are also included. The regression equation error follows a standard normal distribution. Except for H0, the coefficients of each model are multiplied by a common constant so as to make the theoretical $R^{2}$ equal to 0.75 . Theoretical $R^{2}$ is defined as

$$
\text { Theoretical } R^{2}=\frac{\left(\boldsymbol{\mu}-\mu_{0} \mathbf{1}\right)^{T}\left(\boldsymbol{\mu}-\mu_{0} \mathbf{1}\right)}{\left(\boldsymbol{\mu}-\mu_{0} \mathbf{1}\right)^{T}\left(\boldsymbol{\mu}-\mu_{0} \mathbf{1}\right)+n \sigma^{2}} \text {, }
$$

where $\sigma^{2}$ is the error variance, $\mathbf{1}$ is a vector of 1 's, and $\mu_{0}=\boldsymbol{\mu}^{T} \mathbf{1} / n$.

For a given combination of $n$ and $\rho$, we generate 100 data sets independently based on the same design matrix and coefficients. All five variable selection methods are applied to each data set. The true model is fit as well. For each method, the mean is calculated for both the model size and the model error.

We augmented the base simulation study with three additional cases. In the first we took $n=150$ with 42 predictors (the original 21 predictors and their squares), in order to study how the different methods perform in the presence of additional unimportant variables. In the second we set $n=500$ with the original 21 predictors, in order to study the performance of the methods with a larger sample size. In the third, we reran the simulations with $n=150$ observations and 21 predictors adjusting the coefficients so that the theoretical $R^{2}$ equaled $0.35,0.95$ and 0.99 , respectively. The third case allowed us to study the consistency of the methods as $R^{2}$ approaches 


\subsection{Simulation Results}

For the simulations with 21 predictors $(n=150,50)$ and $R^{2}=0.75$, the ratios of model error of Best to model error of NAMS, $I C_{p} / F R$, Minimum $C_{p}$ and LB are calculated for each combination of $n$ and $\rho$, and plotted in Figure 4. The larger the ratio, the better the performance. Note that the relative standard error of the estimates (standard error divided by estimate) in Figure 4 is about 0.23 for H0, 0.12 for H1, and 0.06 for H2-H4 and FULL, respectively. Similar relative standard errors are found in the other figures. We did not see much difference between the simulation result with 150 observations and that with 50 observations, except that all the methods have poorer performance when the sample size is smaller. Therefore, we concentrate on the simulation result with 150 observations. Figure 4 shows that NAMS is the best in terms of model error when the true model is H0, H1, H2, or H3. For FULL, NAMS loses to all the other methods. For H4, NAMS is still the best when the predictors are uncorrelated, but as the correlation increases, NAMS underfits and loses to the other methods. In addition, NAMS is the most stable in relative performance with respect to Best, as indicated by its relatively flat performance curve. $I C_{p} / F R$, Minimum $C_{p}$ and LB provide better relative performance as the true model increases in size; however, they do so at the expense of overfitting models of small and moderate sizes. This claim is supported by Figure 5, where the ratios of model size of NAMS, $I C_{p} / F R$, Minimum $C_{p}$ and LB to model size of True are plotted for each combination of $n$ and $\rho$. Figure 5 shows that NAMS always selects the most parsimonious models, while LB and Minimum $C_{p}$ select much larger models especially for H0-H2. Therefore, NAMS is the best in achieving dimension reduction.

For the simulation with 42 predictors, 150 observations, and $R^{2}=0.75$, the ratios of model

error of Best, to model error of NAMS, $I C_{p} / F R$, Minimum $C_{p}$ and LB, are plotted in Figure 6 

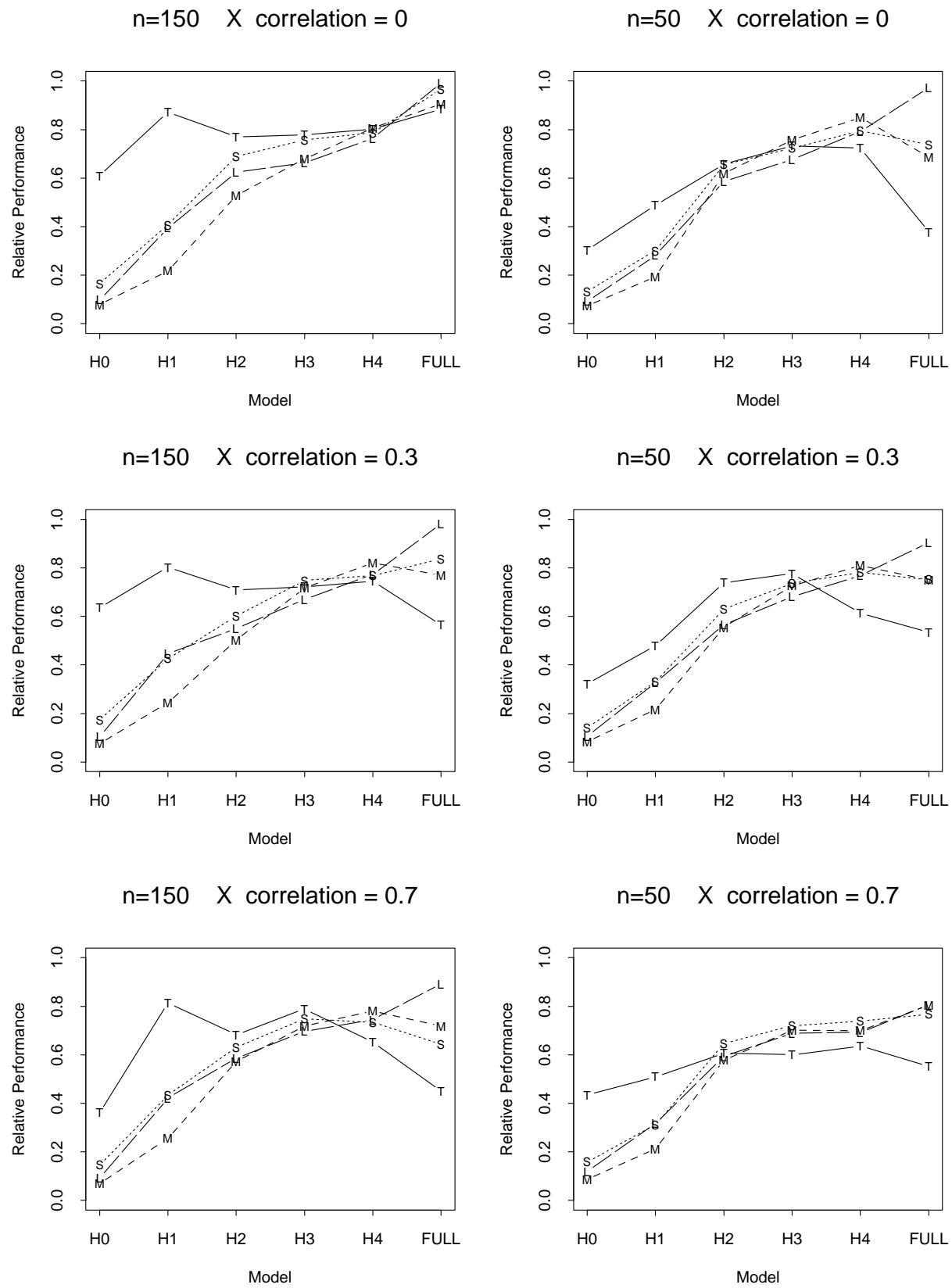

T: NAMS S: $I C_{p} / F R \quad$ M: Minimum $C_{p} \quad$ L: LB

Figure 4: Relative Performance with Respect to Best (21 predictors).

The ratios of model error of Best to model error of NAMS, $I C_{p} / F R$, Minimum $C_{p}$ and LB. 

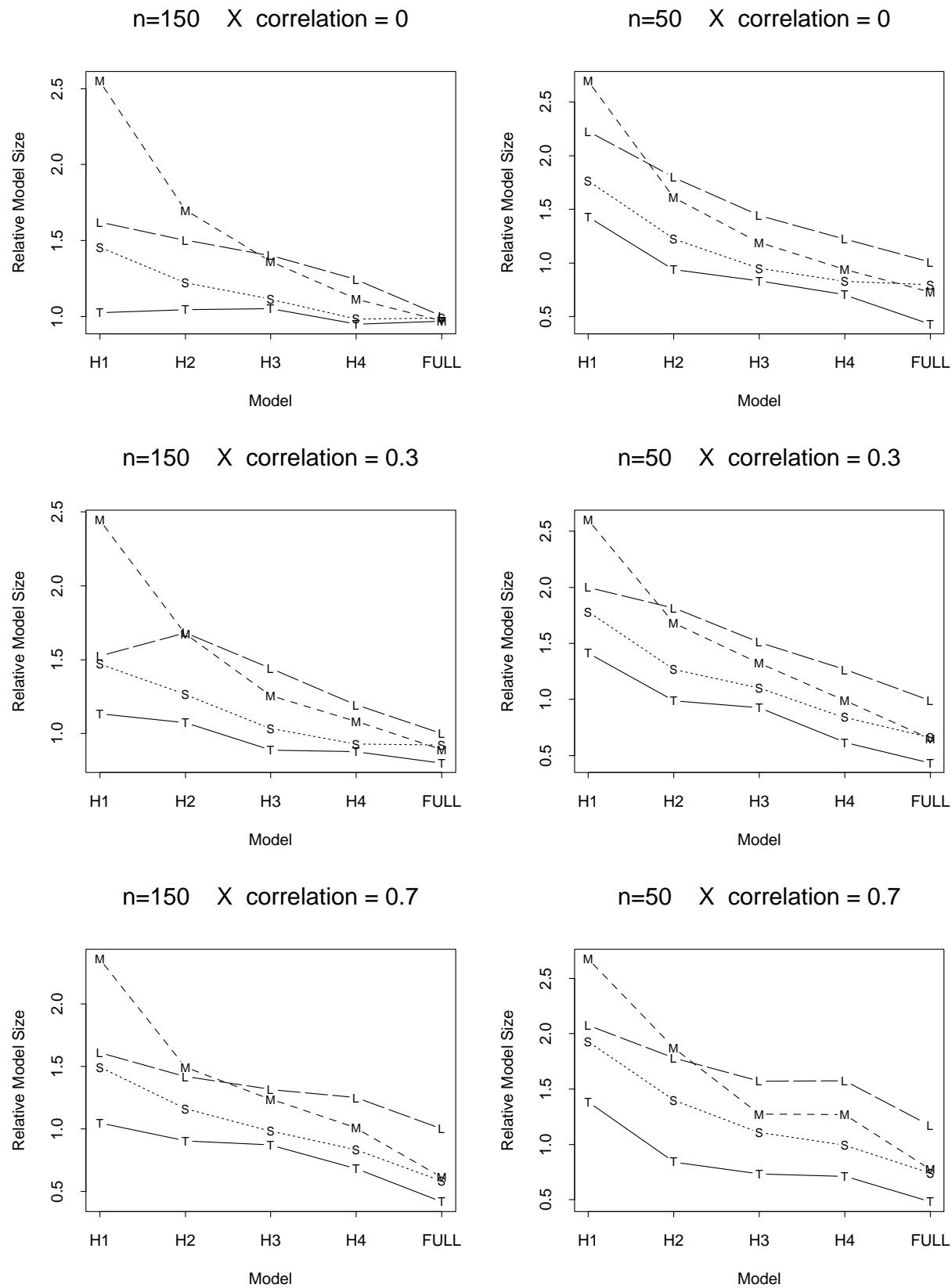
T: NAMS
S: $I C_{p} / F R$
M: Minimum $C_{p}$
L: LB

Figure 5: Relative Model Size with Respect to Best (21 predictors). The ratios of model size of NAMS, $I C_{p} / F R$, Minimum $C_{p}$ and LB to that of Best. 
for each $\rho$. NAMS has the smallest model error for H0-H4 regardless of $\rho$, and for FULL when $\rho=0,0.3$. Moreover, NAMS does not lose as much to the other methods as in the simulation with 21 predictors on FULL with $\rho=0.7$. The other three methods are adversely affected by the additional 21 predictors, and their model errors increase substantially as the number of predictors increases from 21 to 42. However, NAMS is less affected by the additional predictors and its model error does not increase as much as the number of predictors increases from 21 (Figure 4 ) to 42 (Figure 6). It is evident in Figure 6 that NAMS is the overall method of choice for $n=150$ and 42 predictors.

Figure 7 displays results from the simulation with $n=500$ observations, $R^{2}=0.75$ and the original 21 predictors. All methods have much smaller model errors compared to the simulation results with 150 observations, and they are closer to True and Best. Because overfitting is not penalized as much when the sample size is large, NAMS does not outperform the other three methods as much on H0-H3. However, for H4 and FULL, NAMS is nearly as good as the others, except for FULL with $\rho=0.7$.

Finally, the simulation with different $R^{2}$ showed that as $R^{2}$ approaches 1 , the model error of all methods approached 0. This experiment, whose results are not displayed in this paper, used the same design matrix and error for all $R^{2}$. We adjusted the coefficients instead of the error variance, to get the desired theoretical $R^{2}$.

\subsection{Conclusion and Discussion}

From the simulation with 150 observations and 21 predictors (Figures 4, 5), we see that in terms of model error, NAMS is the best when the true model is simple (H0-H2). It is marginally better than the other methods when the true model is H3. It provides the best prediction by using the fewest variables, and thus is the best in achieving dimension reduction. NAMS loses to the 

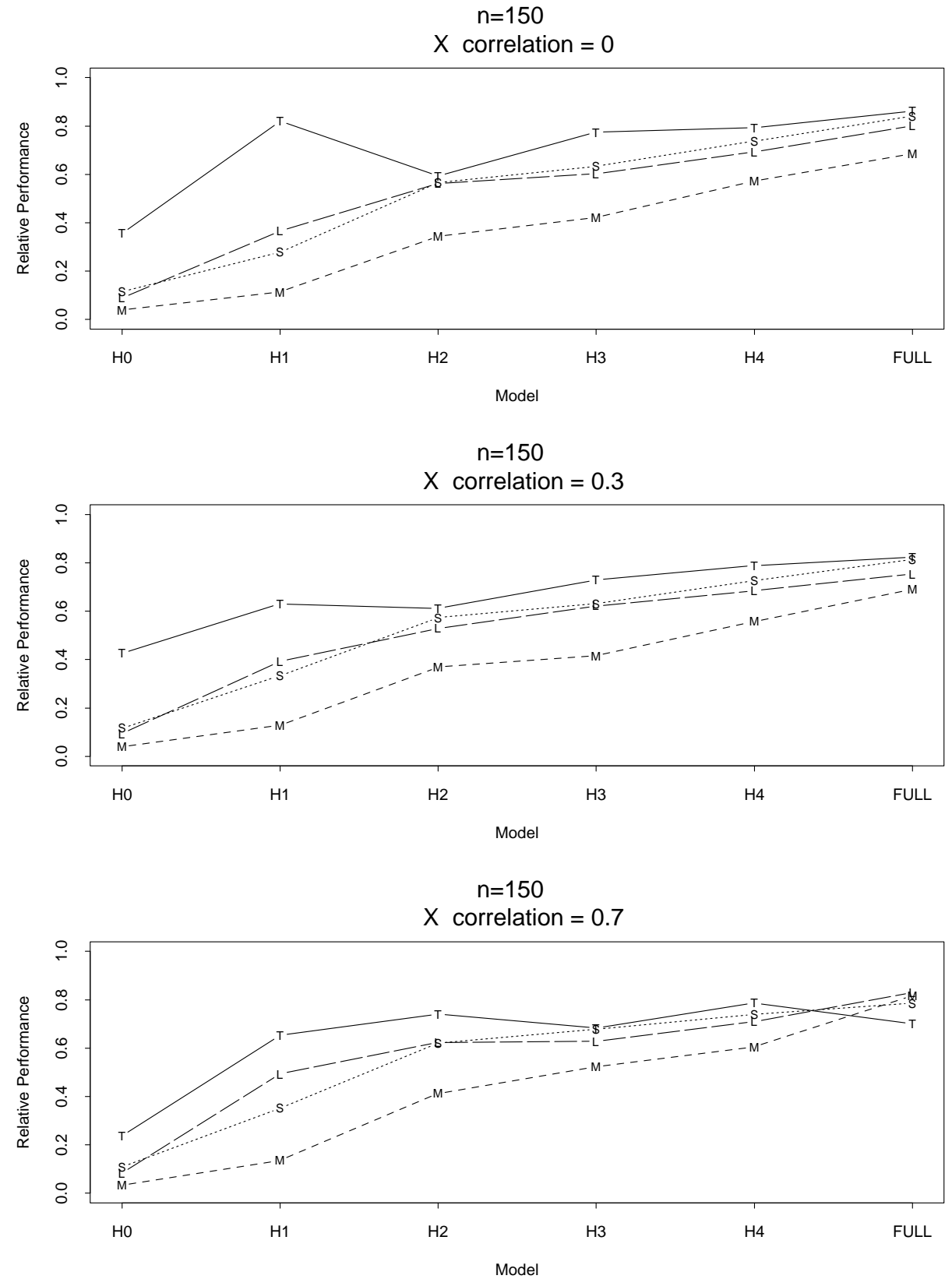

T: NAMS S: $I C_{p} / F R \quad$ M: Minimum $C_{p} \quad$ L: LB

Figure 6: Relative Performance with Respect to Best (150 observations and 42 predictors). The ratios of model error of Best to model error of NAMS, $I C_{p} / F R$, Minimum $C_{p}$ and LB. 

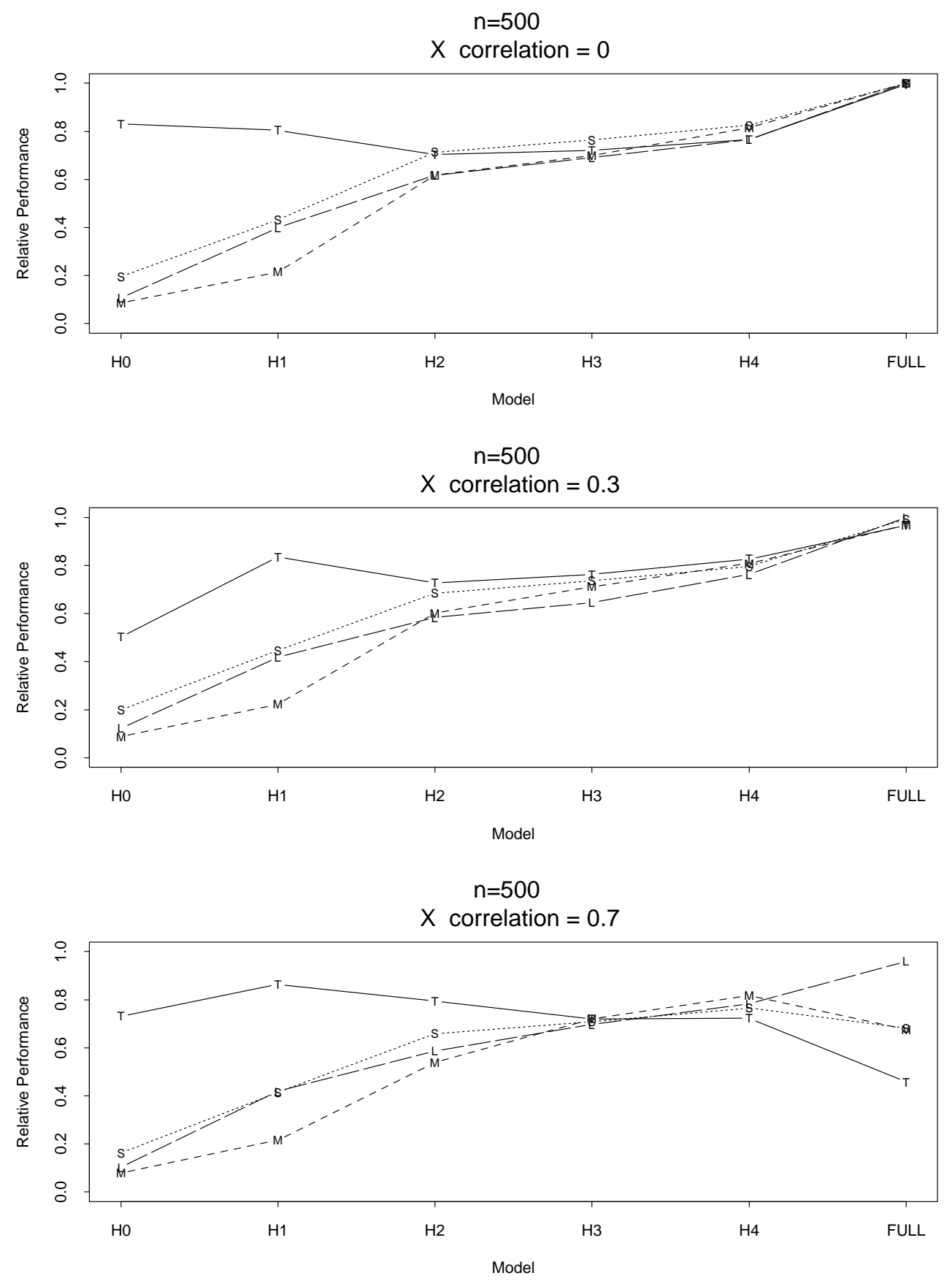

T: NAMS S: $I C_{p} / F R \quad$ M: Minimum $C_{p} \quad$ L: LB

Figure 7: Relative Performance with Respect to Best (500 observations and 21 predictors). The ratios of model error of Best to model error of NAMS, $I C_{p} / F R$, Minimum $C_{p}$ and LB. 
others if the true model is complicated (H4 or FULL), especially when the correlation between the predictors is high. But as shown in Figure 4, NAMS is the most stable in relative performance with respect to Best, while $I C_{p} / F R$, Minimum $C_{p}$ and LB tend to overfit. Because they overfit, these three methods provide better relative performance as the model gets more complicated. On the whole, Minimum $C_{p}$ overfits badly and provides very poor performance and is not recommended in practice. $I C_{p} / F R$ is better than Minimum $C_{p}$ except for $\mathrm{H} 4$.

We see a similar pattern in the simulation with 50 observations and 21 predictors, except that every method performs worse due to the small sample size. When the sample size is 50 , the ratio of the number of predictors to the number of observations is greater than 0.25 so that it is difficult to find a satisfactory model (Freedman et al. 1992).

For 500 observations and 21 predictors (Figure 7), all of the methods perform better, especially for H4 and FULL, and all have similar model errors in most cases. However, Minimum $C_{p}$ overfits badly for H0-H2. NAMS has the smallest model error for H0-H2. There is not much difference between the four methods for H3, H4 and FULL, except that NAMS loses to the other methods for FULL with $\rho=0.7$. We also recorded average mean squared errors (MSE) of the models selected by the different methods. With the exception of FULL, $I C_{p} / F R$, Minimum $C_{p}$ and LB always had Average MSE $<1$ (= the true error variance), indicating that these methods tended to overfit thereby underestimating residual variation. NAMS unbiasedly estimated error variance in all cases except for FULL.

The simulation with 150 observations and 42 predictors (Figure 6) shows that NAMS successfully ignores the additional unimportant variables and outperforms the other methods except for the FULL model when $\rho=0.7$. The other three methods frequently fit the unimportant variables, resulting in inflated model errors. 
NAMS has the best prediction ability when the true model is simple. For complicated models with greater correlation in the design matrix, NAMS loses to the other three methods in terms of model error due to its tendency to underfit; but NAMS is as good as or better than the other methods if either the sample size or the theoretical $R^{2}$ is large. In addition, NAMS provides much better performance than any of the other three methods when the number of predictors is large, except for FULL with $\rho=0.7$. In summary, NAMS is good at finding a parsimonious model without sacrificing prediction ability when such a model exists.

\section{EXAMPLES}

We now illustrate the method on two data sets.

Pollution Data. (McDonald and Schwing 1973). This data set has 60 observations and 15 predictors. The response variable is the Total Age Adjusted Mortality Rate obtained for the years 1959 - 1961 for 201 Standard Metropolitan Statistical Areas (SMSA). The result from ridge regression is copied from McDonald and Schwing (1973). We apply NAMS, LB, $I C_{p} / F R$, Minimum $C_{p}$, and Intelligent $C_{p}$ to the data. The results are summarized in Table 1. All the methods have similar performance in terms of model size and $R^{2}$. NAMS, $I C_{p} / F R$ and $I C_{p}$ select the same five-variable model. LB and Minimum $C_{p}$ select the same six-variable model, and a different sixvariable model was chosen by McDonald and Schwing (1973) using ridge regression. For NAMS, $\alpha_{0}$ is estimated to be 0.03 which corresponds to a five-variable model $(1,2,6,9,14)$. Figure 8 (left panel) displays the slope-versus- $\alpha$ plot from which the optimal choice of $\alpha$ was obtained.

Rubber Data. (Lin 1993). The original data set in Williams (1968) contains 28 runs and 23 predictors (there were 24 predictors in the paper, but two of them are identical). Lin (1993)

took half of the data set (14 runs and 23 predictors) to demonstrate the use of supersaturated (SSD) designs, as shown in Table 3 of Lin (1993). Forward selection was employed by Lin (1993) 
Table 1: Pollution Data

\begin{tabular}{|l||c|c|c|c|}
\hline Method & Variables Selected & Model Size & $R^{2}$ & Number of Resamples \\
\hline Ridge Regression* & $1,2,6,8,9,14$ & 6 & 0.724 & - \\
\hline NAMS & $1,2,6,9,14$ & 5 & 0.717 & 4000 \\
\hline LB & $1,2,3,6,9,14$ & 6 & 0.735 & 100 \\
\hline$I C_{p} / F R$ & $1,2,6,9,14$ & 5 & 0.717 & - \\
\hline Minimum $C_{p}$ & $1,2,3,6,9,14$ & 6 & 0.735 & - \\
\hline$I C_{p}$ & $1,2,6,9,14$ & 5 & 0.717 & - \\
\hline
\end{tabular}

* From McDonald and Schwing (1973)

Table 2: Rubber Data (Williams 1968)

\begin{tabular}{|l||c|c|c|}
\hline Method & Model Size & $R^{2}$ & Number of Resamples \\
\hline Lin (1993) & 5 & 0.973 & - \\
\hline Westfall, Young and Lin (1998) & 1 & 0.632 & 200000 \\
\hline NAMS & 1 & 0.632 & 4000 \\
\hline LB & 8 & 0.999 & 100 \\
\hline$I C_{p} / F R$ & 8 & 0.999 & - \\
\hline Minimum $C_{p}$ & 8 & 0.999 & - \\
\hline$I C_{p}$ & 8 & 0.999 & - \\
\hline
\end{tabular}

to analyze the data and "the important factors were identified as 15, 12, 20, 4, and 10 with an $R^{2}=97.3 \%$." As pointed out by Wang (1995), factors 10 and 15 do not show up even in a model with $R^{2}=0.99$ when forward selection is applied to the other half of the data set. Later, Westfall, Young and Lin (1998) attributed this inconsistency to multiple testing and developed a resamplingbased method to adjust the p-value for multiplicity. As shown in their Table 3, only one variable (factor 15; adjusted p-value 0.016) is selected, because the next candidate variable, factor 12, has an adjusted p-value of 0.816 , and is deemed as non-significant.

The mean squared error from the full model provides the reference line used by the NAMS method to tune forward selection. Thus NAMS is not directly applicable to super saturated designs (SSD). However, such designs are commonly used under the assumption of effect sparsity, i.e., when only a few of the many predictors are thought to be important. In such cases a liberal, initial model fit resulting in a high $R^{2}$ will likely include all of the important predictors. This suggests a two-stage 

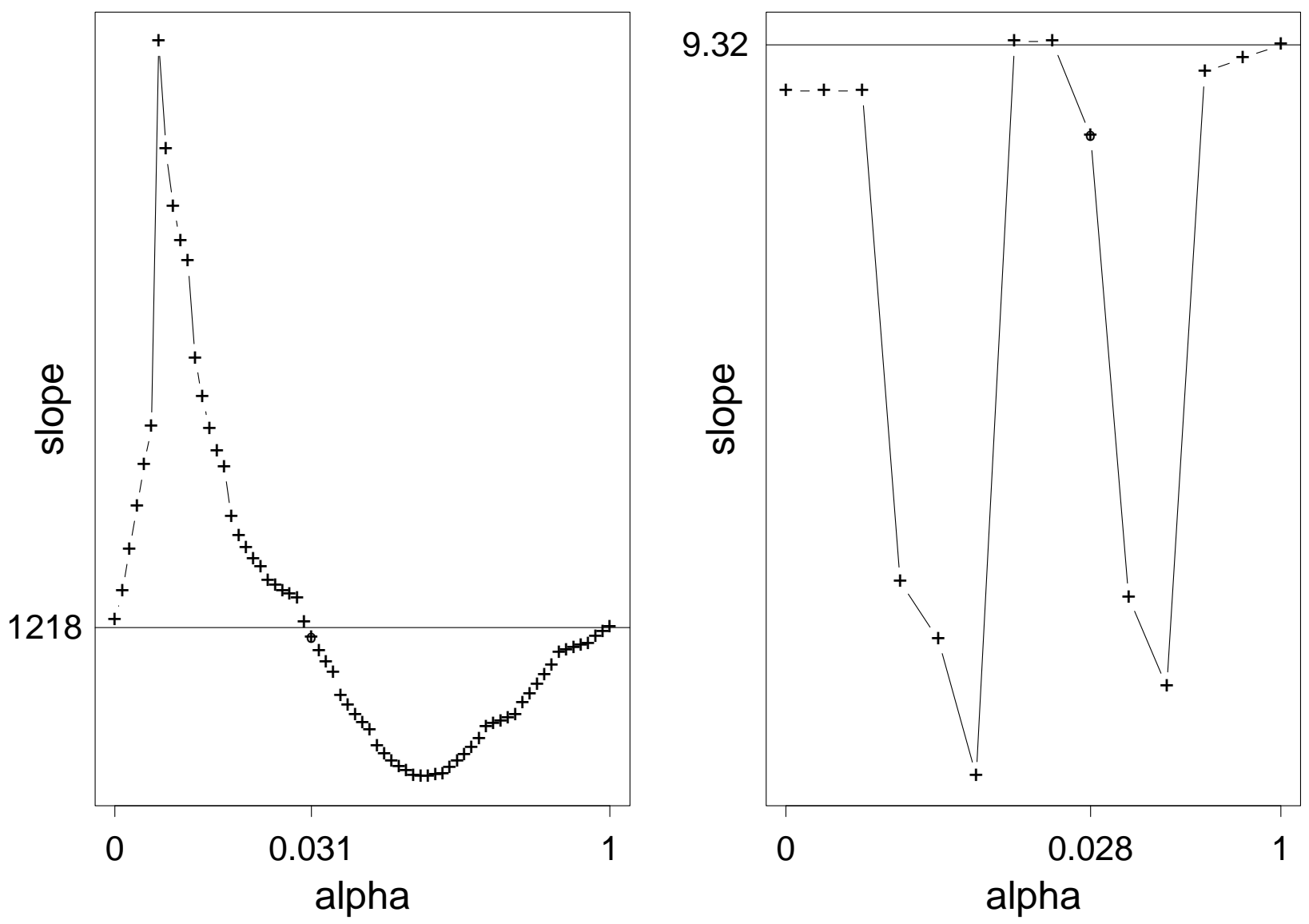

Figure 8: Slope-versus-alpha plots for the real data examples. Circled point $\oplus$ indicates optimal $\alpha$. Left panel, Pollution data; right panel, Rubber data.

adaptation of NAMS for SSDs. In the first stage use a liberal model fitting strategy to obtain a collection of predictors resulting in a large $R^{2}$. Then starting with the predictors so identified, use NAMS to pare down the set of predictors. We now show that this two-stage approach produces a reasonable model for the Rubber data.

In the Rubber data, the model with 8 predictors from forward selection has an $R^{2}=1.000$. So, we concentrate on these 8 predictors. NAMS, LB, $I C_{p} / F R$, Minimum $C_{p}$ and Intelligent $C_{p}$ were applied to select models. As shown in Table 2, NAMS selects the one-variable model with $\alpha_{0}$ estimated to be 0.028 , while $\mathrm{LB}$, Minimum $C_{p}, I C_{p}$, and $I C_{p} / F R$ all select an eight-variable model, i.e. the full model. Figure 8 (right panel) displays the slope-versus-alpha plot for the Rubber data. 
We also applied the five methods using pilot studies with the first 3, 4, 5, 6, and 7 predictors selected in forward selection, respectively, and obtained very consistent results: NAMS always selects the one-variable model, while the other methods always select the largest possible model.

As another way to evaluate the methods, we applied them to the original data set (28 observations and 23 predictors). $\mathrm{LB}, I C_{p}$, and $I C_{p} / F R$ select a three-variable model, and Minimum $C_{p}$ selects a five-variable model (note that the predictors enter the model in a different order in the original data). NAMS again selects the same one-variable model (factor 15), while the other methods apparently overfit the data.

\section{REFERENCES}

Atkinson, A. C. (1980), "A Note on the Generalized Information Criterion for Choice of a Model," Biometrika, 67, 413-418.

Breiman, L. (1992), "The Little Bootstrap and Other Methods for Dimensionality Selection in Regression: X-Fixed Prediction Error," J. Am. Stat. Assoc., 87, 738-754.

Carroll, R. J., Ruppert, D. and Stefanski, L. A. (1995), Measurement Error in Nonlinear Models, London: Chapman Hall.

Cook, J. R. and Stefanski, L. A. (1994), "Simulation-Extrapolation Estimation in Parametric Measurement Error Models," J. Am. Stat. Assoc., 89, 1314-1328.

Draper, Norman R. and Smith, Harry (1981), Applied Regression Analysis (Second Edition), New York: John Wiley \& Sons.

Freedman, L. S., Pee, D., and Midthune, D. N. (1992), "The Problem of Underestimating the Residual Error Variance in Forward Stepwise Regression," Statistician, 41, 405-412.

Lin, D. K. J. (1993), “A New Class of Supersaturated Design,” Technometrics, 35, 28-31. 
Mallows, C. L. (1973), "Some Comments on $C_{p}$," Technometrics, 15, 661-675.

Mallows, C. L. (1995), "More Comments on $C_{p}$ " Technometrics, 37, 362-372.

McDonald, G. C. and Schwing, R. C. (1973), "Instabilities of Regression Estimates Relating Air Pollution to Mortality," Technometrics, 15, 463-481.

Shen, X. and Ye, J. (2002), "Adaptive Model Selection," Journal of the American Statistical Association 97, 210-221.

Stefanski, L. A. and Cook, J. R. (1995), "Simulation-Extrapolation: The Measurement Error Jackknife," Journal of the American Statistical Association 90, 1247-1256.

Tibshirani, R. and Knight, K. (1999), "The Covariance Inflation Criterion for Adaptive Model Selection," J. R. Statist. Soc., 61, 529-546.

Wang, P. C. (1995), "Letters to the Editor: Comments on Lin (1993)," Technometrics, 37, $358-359$.

Westfall, P. H., Young, S. S. and Lin, D. K. J (1998), "Forward Selection Error Control in the Analysis of Supersaturated Designs," Statistica Sinica, 8, 101-117.

Williams, K. R. (1968), "Designed Experiments," Rubber Age, 100, 65-71.

Ye, J. (1998), "On Measuring and Correcting the Effects of Data Mining and Model Selection," J. Am. Stat. Assoc., 93, 120-131.

Zhang, P. (1992), "On the Distributional Properties of Model Selection Criteria," J. Am. Stat. Assoc., 87, 732-737. 